ISSN: 0212-0267

DOI: http://dx.doi.org/Io.I420I/hedu20I837199222

\title{
LA DISIDENCIA SEXUAL Y LA EDUCACIÓN COMO ACTIVISMO
}

\section{Sexual dissidence and education as activism}

\author{
Renée DePalma \\ Universidade da Coruña \\ Correo-e: r.depalma@udc.es \\ Inés Cebreiro López \\ Universidade da Coruña \\ Correo-e: inescebreirolopez9o@gmail.com
}

Recepción: 4 de julio de 20I7. Envío a informantes: 20 de julio de 2017

Aceptación definitiva: I de octubre de 2017

REsumen: En este artículo, analizamos las varias líneas de activismo relacionadas con la diversidad afectivo-sexual y de género, así como el impacto que tuvieron, y que siguen teniendo, en los ámbitos educativos. Analizamos aquí estos movimientos en dos grandes bloques. Primero, revisaremos el activismo LGBT, movimiento que ha abogado por la igualdad y visibilidad de las personas gais, lesbianas, transexuales y bisexuales. Más adelante, identificaremos también otros movimientos más radicales, basados en líneas de pensamiento transfeministas, que se resisten a la propia noción de normal extendiendo las críticas hacía el patriarcado del feminismo radical, al incluir entre ellas la propia construcción social del binarismo sexual y de género. Así mismo, revisamos algunos esfuerzos realizados por parte de colectivos de activistas para educar sobre la diversidad afectivo-sexual y de género, dado que identificamos el ámbito escolar como espacio privilegiado tanto para reproducción de ideologías sociales erróneas y dañinas como para la resistencia ante las mismas.

Palabras Clave: LGBT; intersex; transfeminismo; queer/cuir; diversidad afectivo-sexual y de género; movimientos sociales; escuela.

АвSTRACT: In this article, we analyze several lines of activism related to gender and sexual diversity, along with the impact they have had, and continue to have, in school contexts. We analyze these movements along two main lines - first we 
review LGBT activism, movements that have promoted equality and visibility of gay, lesbian, transsexual and bisexual persons. At the same time, we consider other more radical movements based on transfeminist thinking that resist the very notion of normal, extending feminist critique of the patriarchy to include the social construction of the sexual binary. We identify the institution of schooling as a key site for disseminating inaccurate and harmful social ideologies, as well as for their resistance. We review here some of the efforts of activist collectives to educate about gender and sexual diversity.

KEY WORDS: LGBT; intersex; transfeminism; queer; gender and sexual diversity; social movements; schooling.

\section{Introducción}

$\mathrm{E}$ N 20ir, la Unión Europea, en el artículo 2i de su Carta de Derechos Fundamentales, censura legalmente la discriminación por motivos de orientación sexual: «Se prohíbe toda discriminación, y en particular la ejercida por razón de sexo, raza, color, orígenes étnicos o sociales, características genéticas, lengua, religión o convicciones, opiniones políticas o de cualquier otro tipo, pertenencia a una minoría nacional, patrimonio, nacimiento, discapacidad, edad u orientación sexual» ${ }^{\mathrm{I}}$. Este mismo año, el Consejo de Derechos Humanos de la ONU aprobó la resolución I7/I9, la primera en la que se reconocen los derechos del colectivo LGBT, y una declaración formal de condena de los actos de violencia y discriminación en cualquier lugar del mundo por razón de la orientación sexual e identidad de género².

Leila Rupp considera que estos logros legislativos hicieron realidad los retos establecidos unos 60 años antes, con la formación en I95I de la Comisión Internacional para la Igualdad Sexual (ICSE), representada por países como Dinamarca, Alemania Occidental, Gran Bretaña, Italia, Holanda y Suiza. En su petición a la ONU, reclamaron la extensión de los derechos humanos a «minorías homosexuales» de acuerdo con «los hallazgos de la investigación psicológica, biológica, y médica» y la «conciencia aumentada de la justicia social por parte de la humanidad»-esto último era una referencia a las atrocidades nazis durante la II Guerra Mundial ${ }^{3}$. La ICSE consolidó el ascendente movimiento homófilo, siendo precursora de la liberación homosexual, que adquirirá un tono más radical y claramente relacionado con la política de la(s) identidad(es) no-heterosexuales. Estos antecedentes homófilos adoptaron nombres sin referencias específicas a la homosexualidad, como «La Sociedad para Derechos Individuales» (San Francisco, I964), o con referentes obscuros, como «La Sociedad Mattachine» (Los Angeles, 1950).

http://ec.europa.eu/justice/fundamental-rights/homophobia/index_en.htm.

«Born free and equal: Sexual Orientation and Gender Identity in International Human Rights Law», HR/PUB/r2/o6, New York, United Nations, 2012.

3 Rupp, Leila: «The Persistence of Transnational Organizing: The Case of the Homophile Movement», The American Historical Review, II6, n. ${ }^{\circ} 4$ (20II), pp. IOI4-IO39. 
En las décadas de 1960 y 1970 se irá abriendo paso la nueva perspectiva de liberación homosexual, en la que pueden diferenciarse dos momentos importantes de ruptura: primero, con la aparición de la lucha bajo el lema de gay power, el empoderamiento homosexual, que buscaba una transformación más completa y radical de la sociedad; y después con un enfoque más específicamente centrado en la afirmación de una identidad homosexual'.

Con el paso de tiempo, estos colectivos empiezan a compartir el escenario con movimientos alternativos, que buscan desafiar la creación y mantenimiento de lo normal y rechazan políticas de inclusión y tolerancia. El enfoque identitario, inicialmente conceptualizado como una plataforma de reivindicación radical y transformación social, empieza a ser criticado por limitarse a reclamar la mera inclusión en un sistema heteropatriarcal y por excluir experiencias menos inteligibles o socialmente aceptables. Nos sirve de ejemplo el colectivo chileno cuds (Colectivo Universitario de Disidencia Sexual), que define su trabajo como la «irrupción de los imaginarios sexuales normativos»s. Son varios los colectivos que señalan los centros educativos en particular como espacios politizados que promueven el generismo ( «la creencia generalizada y sistémica en la naturalidad y en la superioridad de la generonormatividad» $)^{6}$ y la heteronormatividad, donde «la heterosexualidad se posiciona como la categoría por defecto de la sexualidad y, por tanto, se normaliza» ${ }^{7}$.

En este artículo analizamos las varias líneas de activismo relacionadas con la diversidad afectivo-sexual y de género, y el impacto que tuvieron, y que siguen teniendo, en los ámbitos educativos. Para ello los clasificaremos en dos bloques. Primero revisaremos el activismo LGBT, movimiento que ha abogado por la igualdad y visibilidad de las personas gais, lesbianas, transexuales y bisexuales. Es cierto que, en muchos casos, los derechos de personas que se identifican como intersexual, transexual o cuyas experiencias se definen como queer por no acoplarse fácilmente a una categoría de sexo o sexualidad están incluidos en estos colectivos, por lo menos simbólicamente por la adición de siglas (LGBTIQ...). $)^{8}$ Sin embargo, hemos decidido tratarlos de forma separada en nuestro análisis, porque aportan visiones y posibilidades educativas más en línea con los movimientos postidentitarios. Nuestro análisis se centrará en el Estado español, haciendo referencias a la situación internacional para establecer puntos de inspiración o comparación.

4 Armstrong, Elizabeth: Forging Gay Identities: Organizing Sexuality in San Francisco, 19501994, Illinois, University of Chicago Press, 2002.

https://disidenciasexualcuds.wordpress.com/about/.

6 Airton, Liz: «From sexuality (gender) to gender (sexuality): The aims of anti-homophobia education», Sex Education, 9, n. ${ }^{\circ} 2$ (2009), p. 132.

WaLton, Gerald: «Eating cake: The paradox of sexuality as a counter-diversity discourse», en Steinberg, Shirley R. (ed.): Diversity and multiculturalism: a reader, New York, Peter Lang, 2009, p. 212, http://hdl.library.upenn.edu/I0I7.12/366263.

El Gobierno de Australia utiliza el término LGBTI (ver http://lgbtihealth.org.au/lgbti/) y la asociación de Servicios para Niños de Nueva York (EE. UU.) utiliza LGBTQ en su documentación (ver https://wwwi.nyc.gov/site/acs/about/lgbtq-support.page). 


\section{La emergencia del activismo LGBT}

Antes de empezar a revisar la historia del activismo LGBT, quizás conviene reconocer la opresión sistemática sufrida por generaciones de «disidentes sexuales» antes de gozar de reconocimiento o representación. Podemos resaltar una pequeña mejora en 1567 , cuando la incineración viva pasó de ser un método probatorio a constituir una pena aplicable a los sujetos considerados culpables de sodomía o hermafrodismo, una condena para la que «bastaba con un simple testimonio para evidenciar la comisión de tal delito, aunque dicho testimonio entrara en contradicción con otros»? .

Según Vázquez García, los conceptos de sexo, género y sexualidad no conseguirán disociarse hasta los años I950, y, por lo tanto, las divergencias en términos de deseos, corporalidades y comportamientos fueron consideradas en su conjunto como aberraciones o desafíos del orden natural ${ }^{10}$. Su castigo dependía de varios factores sociopolíticos. Este mismo autor contrasta, por ejemplo, la reacción institucional ante Helena de Céspedes (Granada, 1546) con la inspirada por Catalina de Erauso (San Sebastián, I592). La primera, esclava emancipada e hija de esclavos moriscos, fue condenada por el tribunal inquisitorial de Toledo a una sentencia de doscientos azotes y diez años de reclusión, a pesar de su reconocido servicio militar. La segunda, aun siendo responsable de por lo menos in asesinatos cometidos en altercados varios, amante de múltiples mujeres e incluso haberse casado al menos con una de ellas, se convirtió en "un personaje popularísimo y, simultáneamente, en paradigma de caballero español perfecto y de la dama española ejemplar» ${ }^{\mathrm{II}}$. Aunque la noción de interseccionalidad fue introducida por feministas negras unos 400 años más tarde ${ }^{\mathrm{I} 2}$, podemos ver en estas experiencias históricas como «la sexualidad atraviesa y co-constituye otras formas de desigualdad» ${ }^{13}$.

Volviendo a situarnos en el plano internacional y en la Edad Moderna, el inicio del movimiento LGBT está asociado en la memoria colectiva con las Revueltas de Stonewall en Nueva York. La resistencia a una inesperada redada policial en la noche del 28 de junio de 1969, protagonizada en gran medida por jóvenes indigentes que dormían en un parque cercano y consideraban el bar como lo más parecido que tenían a un hogar, convirtió el Stonewall Inn en el símbolo de una revolución. No obstante, cabe mencionar que los disturbios de agosto de 1966 del café Compton en San Francisco preceden a este acontecimiento, y tal vez no es del todo casual que fueran protagonizados por mujeres transexuales que a

9 García López, Daniel J.: «Politización de la vida y medicalización de la política: la producción del cuerpo intersexual», Eikasia. Revista de Filosofía, 75 (2017), p. 144.

io Vázquez García, Francisco: «¿Por qué en la Edad Moderna no podía haber transexuales? Cuatro casos de transmutación sexual en España (siglos XvI-Xx)", UbiSunt. Revista de Historia, 26 (20II), pp. 49-58.

"I Ibidem, p. 55 .

I2 Crenshaw, K: «Mapping the Margins: Intersectionality, Identity Politics, and Violence against Women of Color», Stanford Law Review, 43, n. 6 (199I), pp. I24I-I299.

${ }^{13}$ Platero Méndez, Raquel (Lucas): Intersecciones: cuerpos y sexualidades en la encrucijada, Barcelona, Bellaterra, 20I2, p. I6. 
su vez fueron excluidas de los pubs frecuentados por clientela homosexual. De hecho, activistas trans tuvieron una participación notable en la emergencia de los movimientos «gay» de los Estados Unidos ${ }^{14}$. Prostituta y Dragqueen, Sylvia Rodríguez ha sido miembro fundadora de las formaciones Gay Liberation Front y Gay Activists Alliance en la ciudad estadounidense de Nueva York ${ }^{15}$.

Mientras en América del Norte se iniciaba la lucha por la libertad afectivosexual, en este rincón de Europa seguíamos bajo la dictadura franquista. En 1954 se reformulaba la Ley de Vagos y Maleantes para incluir a los homosexuales en el saco de los «rufianes y proxenetas», «mendigos profesionales» y «los que vivan de la mendicidad ajena» ${ }^{16}$. Un año después de Stonewall, el 4 de agosto de 1970, era sustituida por la Ley sobre Peligrosidad y Rehabilitación Social, que seguía equiparando la homosexualidad a la prostitución, el tráfico de personas, la delincuencia o la toxicomanía, y declarando abiertamente la peligrosidad social de los que calificaba como «marginados de una vida ordenada y normal $»^{17}$. Esta última ley contemplaba la «prohibición de residir en el lugar o territorio que se designe o de visitar ciertos lugares o establecimientos públicos», así como el «internamiento en centros de reeducación» y el «sometimiento a vigilancia» de las trabajadoras y las personas homosexuales; lo que en la práctica se traducía en internamientos en manicomios y penas de cárcel ${ }^{18}$.

Pese a la desproporcionada e injusta represión, la reacción visible en el Estado español se hizo esperar. Tras años organizándose en la sombra, el 26 junio de 1977 el Front d'Alliberament Gai de Catalunya convoca una manifestación multitudinaria. Consiguieron que 4.000 personas inundasen la Rambla de Barcelona bajo el lema «Nosotros no tenemos miedo, nosotros somos» y reclamasen amnistía para los delitos sexuales y la derogación de la Ley de Peligrosidad y Rehabilitación Social ${ }^{19}$.

Este objetivo se alcanzará de forma parcial en 1979, con la eliminación del artículo referente a los actos de homosexualidad, pero a pesar de ello las fuerzas de la ley y el orden persistían en sus prácticas discriminatorias a través de redadas, cierre de locales de ambiente, etc. Por si no resultaba suficiente, en la década de I980 el VIH se da a conocer, convirtiéndose rápidamente en motivo de alarma social. Como nos explica Guasch, el viH no hace más que reproducir el esquema

14 STRYKer, Susan: «Transgender History, Homonormativity, and Disciplinarity», Radical History Review, n. ${ }^{\circ}$ IOO (2008), pp. I45-157, https://doi.org/I0.I215/01636545-2007-026.

is Su autobiografía relativa a los primeros tiempos de activismo «gay» puede leerse en Rivera, Sylvia: «Queens in Exile, the Forgotten Ones», en Nestle, Joan; Howell, Clare y Wilchins, Riki Anne (eds.): GenderQueer: Voices from beyond the Sexual Binary, Los Angeles, Alyson Books, 2002, pp. 67-85.

${ }_{16}$ Boletín Oficial del Estado, n. ${ }^{\circ} 198,17$ de julio de 1954.

17 Boletín Oficial del Estado, n. ${ }^{\circ}$ I87, 6 de agosto de 1970.

18 Cerca de un millar de personas cumplieron condena por su orientación sexual durante los nueve años de vigencia de esta ley (véase Conde, Jesús: «La cárcel de homosexuales de Badajoz: cuando el franquismo castigaba a los que amaban libres», El Diario, 03-07-2016).

19 Bernardo, Ángela: «Homosexualidad en España, la historia del movimiento LGTB», Hipertextual, 28 de junio de 20I5, https://hipertextual.com/20I5/06/homosexualidad-en-espana-historia. 
antiguo que percibe la enfermedad «como una consecuencia de la transgresión de la norma efectuada por el propio grupo social (nuestros pecados, el libertinaje sexual, la impiedad) o bien como consecuencia de una agresión ajena al grupo (extranjeros, marginados, judíos, herejes, gays) ${ }^{20}$. En la percepción social, ante la «epidemia» del sIDA, la homosexualidad servirá tanto para representar el mal de dentro como el de fuera.

Solamente quince años después, con la aprobación del Código Penal de 1995 , se establece a nivel legal la necesidad de protección de la orientación sexual y la homofobia como agravante de otros delitos ${ }^{21}$. De pronto, en 2005 se da un paso de gigante. Tras años de luchas sin grandes victorias, se aprueba la Ley i3/2005, convirtiendo al Estado español en el tercero del mundo en reconocer legalmente el derecho al matrimonio entre personas del mismo sexo ${ }^{22}$.

Dos años después, en 2007, tiene lugar otro pequeño gran avance: la aprobación de la Ley 3/2007 reguladora de la rectificación registral de la mención relativa al sexo de las personas ${ }^{23}$. Esta hace posible que las personas modifiquen los datos referentes al sexo y se registren con un nuevo nombre que concuerde con la identidad sentida, sin necesidad de haberse sometido a cirugía de reasignación. Se exige, eso sí, la presentación de un diagnóstico de disforia de género y acreditar dos años de sometimiento a tratamiento médico «para acomodar sus características físicas a las correspondientes al sexo reclamado», pero este tratamiento no tiene por qué incluir la cirugía, y además «no será un requisito necesario [...] cuando concurran razones de salud o edad que imposibiliten su seguimiento». Se abre así la puerta para que las personas menores de edad puedan modificar legalmente su nombre para que concuerde con el sexo reclamado sin iniciar tratamientos médicos, facilitando de esta forma que las escuelas y otras instituciones les ofrezcan un tratamiento en sintonía con su situación personal.

Pero no solo el Estado español daba grandes pasos, pues en la primera década del siglo xxi la Organización de las Naciones Unidas (ONU), el Parlamento Europeo y la Agencia de Derechos Fundamentales de la Unión Europea (FRA) aprueban y publican numerosos informes de recomendaciones, cartas de derechos y tratados internacionales legalmente vinculantes. Entre ellos cabe destacar algunos por sus implicaciones para el campo educativo. En 2006, en respuesta a lo que los expertos internacionales en derechos humanos consideraban «patrones bien documentados de abusos», se comenzaron a establecer una serie de principios relativos a la orientación sexual y a la identidad de género. El principio 16 de los llamados Principios de Yogyakarta establece que «toda persona tiene derecho a la educación, sin discriminación alguna basada en su orientación

20 Guasch, Óscar: «Para una sociología de la sexualidad», Reis: Revista Española de Investigaciones Sociológicas, n. ${ }^{\circ} 64$ (1993), p. II8.

${ }_{21}$ Ley Orgánica io/ı995, de 23 de noviembre, del Código Penal, Boletín Oficial del Estado, n. ${ }^{\circ}$ 28, 4 de noviembre de 1995 .

${ }_{22}$ Boletín Oficial del Estado, n. ${ }^{\circ}$ 157, 2 de julio de 2005.

${ }^{23}$ Boletín Oficial del Estado, n. ${ }^{\circ} 65, \mathrm{I} 6$ de marzo de 2007. 
sexual e identidad de género, y con el debido respeto hacia estas»²4. Así mismo, la Unión Europea incluyó la homofobia como una de las áreas de igualdad que se deben abordar en los centros educativos: «Es inevitable que las expresiones de racismo, xenofobia, homofobia y sexismo se reflejen también en las comunidades educativas; el acoso escolar es un problema que varios Estados Miembros identificaron como prioritario» ${ }^{25}$. La Organización Internacional Juvenil y Estudiantil de Lesbianas, Gais, Bisexuales, Transexuales y Queer definió la educación como un ámbito particularmente importante, y publicó directrices para una práctica escolar inclusiva, que incluyen metodologías proactivas de enseñanza. "Cuando la heterosexualidad se percibe como superior, el comportamiento de los individuos se restringe a unos roles de género rígidos, lo que provoca la estigmatización y la discriminación de las personas que se desvían de estas normas» ${ }^{26}$.

Un informe sobre «Homofobia y Discriminación por Motivos de Orientación Sexual e Identidad de Género en los Estados Miembros de la Unión Europea» publicado en 2009 por la Agencia de Derechos Fundamentales de la Unión Europea (FRA) realiza las siguientes recomendaciones:

Dado que la escolarización desempeña un papel tan formativo en la vida de todas las personas, es esencial que se adopten medidas para combatir las actitudes basadas en prejuicios y evitar que causen daños. Estas medidas podrían consistir en:

- La introducción de la temática LGBT en los planes de estudios de los centros escolares con objeto de fomentar la tolerancia y el entendimiento mutuo tanto entre el personal como entre los estudiantes, además de sensibilizar respecto a estructuras familiares no tradicionales. Los materiales didácticos podrían incluir libros para compartir con los padres y ayudar a erradicar estereotipos y explicar la diversidad sexual.

- La adopción en los centros educativos de políticas contra el hostigamiento que tengan expresamente en cuenta la homofobia y la transfobia.

- La formación del profesorado sobre la manera de abordar la temática LGBT en la enseñanza, así como la formación de profesores y asesores escolares sobre el modo de actuar en los casos de hostigamiento homófobo y transfóbico ${ }^{27}$.

24 International Commission of Jurists: «The Yogyakarta Principles: The Application of International Human Rights Law in relation to Sexual Orientation and Gender Identity», 2007, http://www.yogyakartaprinciples.org/.

${ }_{25}$ European Commission: «Schools Policy», 20I2, http://ec.europa.eu/education/schooleducation/policy_en.htm.

26 IGLYO: «Teachers' guide to inclusive education» (The International Lesbian, Gay, Bisexual, Transgender, Queer \& Intersex (LGBTQI) Youth and Student Organisation, 20I5), 9, http://www. iglyo.com/wp-content/uploads/2015/o9/Teachers-Guide.pdf.

${ }_{27}$ Homofobia y discriminación por motivos de orientación sexual e identidad de género en los Estados miembros de la Unión Europea. Informe de sintesis, Luxembourg, Publications Office of the European Union, 2009, p. I3. 
En el siguiente apartado, exploramos algunas realidades de la institución escolar, así como algunas respuestas promovidas o apoyadas en gran medida por colectivos LGBT.

\section{Los centros educativos como lugares clave de represión y resistencia}

En la más reciente de las encuestas realizadas por la Agencia de los Derechos Fundamentales de la Unión Europea (FRA), un $67 \%$ de los participantes no heterosexuales declaró ocultar su orientación sexual en el centro educativo, y un 91 \% recordaba comentarios negativos en el aula acerca de alguna persona que era percibida como $\mathrm{LGBT}^{28}$. En España, un estudio realizado por la Federación Estatal de Lesbianas, Gays, Transexuales y Bisexuales (FELGTB) reveló que un 43\% de los chicos y chicas que sufrieron acoso en el centro educativo consideró la posibilidad del suicidio; un 35\% llegó a concretar planes, y un i7\% trató de llevarlo a cabo: esta tasa triplica el porcentaje de intentos de suicidio entre personas que sufrieron acoso en términos generales ${ }^{29}$. Otro estudio español demostró que ocho de cada diez de los estudiantes observados fueron testigos de insultos relativos a la orientación sexual o a la identidad de género percibidas. Mientras que la mayoría de los participantes pensaban que estos insultos eran inaceptables y exigían algún tipo de intervención, uno de cada cinco estudiantes observados les restaban importancia, argumentando que I) se oyen por doquier, de modo que no son para tanto; 2) no tienen mala intención, o 3) son comentarios inevitables porque hay gente que los merece ${ }^{30}$. Según la guía publicada por el Ministerio de Sanidad, Servicios Sociales e Igualdad, "Abrazar la diversidad», el ejercicio del derecho a la diversidad sexual o de género sigue constituyendo la principal causa de acoso y estigmatización en los centros escolares de Europa y de nuestro Estado. El 25\% del alumnado entre los is y los is años ha sido testigo de agresiones homófobas en sus centros educativos. A pesar de que la mayoría declara ser respetuosa hacia los colectivos LGBT, no actúan ante el acoso a terceros, convirtiéndose así en cómplices del silencio y la invisibilización ${ }^{31}$.

Con respecto al marco político, en el preámbulo de la anterior ley educativa (LOE, 2006) la diversidad afectivo-sexual estaba incluida expresamente como un

28 «EU LGBT survey -European Union lesbian, gay, bisexual and transgender survey- Main results» (Luxembourg: Publications Office of the European Union, 20I4), http://fra.europa.eu/en/ publication/20I4/eu-lgbt-survey-european-union-lesbian-gay-bisexual-and-transgender-surveymain.

«Acoso escolar homofóbico y riesgo de suicidio en adolescentes y jóvenes LGB», 20I2, http:// www.felgtb.org/rs/I74I/din2d6ad-54ec-438b-9358-4483f9e98868/48e/filename/informe-resultadosriesgosuicidio-lgb.pdf.

3o Pichardo Galán, José Ignacio y De Stéfano Barbero, Matías: Diversidad y convivencia en los centros educativos: Una oportunidad educativa, Madrid, Universidad Complutense de Madrid, 20I5, http://presentacionidyc.blogspot.com/.

${ }^{31}$ Pichardo Galán et al:: Abrazar la diversidad: Propuestas para una educación libre de acoso homofóbico y transfóbico, Madrid, Ministerio de Sanidad, Servicios Sociales e Igualdad, 2015. 
área de diversidad ${ }^{32}$. La ley actual (LOMCE, 20I3) contempla la «orientación o identidad sexual» entre los factores de discriminación o acoso escolar que se calificarán como «falta muy grave», pero a diferencia de la anterior ley esta no parece incluir tales diversidades de forma proactiva en el currículo escolar ${ }^{33}$. Queda así en manos de la voluntad, sensibilidad y formación de los educadores introducir esta perspectiva en el día a día de las aulas a través de aquellas áreas y contenidos del currículo relacionados con el desarrollo personal y la educación emocional y afectiva ${ }^{34}$.

Los colectivos LGBT pueden aportar mucho al profesorado en este terreno, desconocido para la mayoría de los docentes. Según la estadounidense Red de Educación Gay, Lesbiana, y Heterosexual (GLSEN), un programa de igualdad relativo a las minorías sexuales puede conceptualizarse como una ampliación y una mejora del currículo actual para incluir en él a las personas LGBT y sus experiencias. Esta organización, que desde 1990 está dedicada exclusivamente a abogar por «la creación de escuelas que son seguras y afirmativas para todo el mundo, independientemente a su orientación sexual o su identidad/expresión de género", incluye entre sus múltiples líneas de acción la investigación, la influencia sobre la legislación y la política educativa, la formación del profesorado y la publicación de propuestas y materiales educativos. Entre sus recursos se cuenta un proyecto curricular basado en entrevistas con personas relacionadas con la historia del movimiento LGBT estadounidense, para compensar la invisibilización de personas LGBT en la historia ysu exclusión sistemática de las instituciones públicas ${ }^{35}$.

En el Reino Unido, la organización Stonewall -cuyo nombre rinde homenaje al mítico pub donde estalló el movimiento de liberación homosexual en los Estados Unidos-, trabaja en el ámbito educativo junto con otros sectores como el de la salud pública o el laboral. También publica estudios de investigación, siendo el más reciente una encuesta realizada a 3.713 jóvenes LGBT de entre II y I9 años sobre sus experiencias escolares ${ }^{36}$.

Su constitución en 1989 fue inspirada por la campaña para derogar el infame artículo 38 del Decreto sobre Gobernanza Local de 1988, que prohibía «la enseñanza sobre la aceptabilidad de la homosexualidad como una relación familiar fingida». Por miedo a las repercusiones, muchos profesores decidieron mantener silencio sobre el tema de la diversidad afectivo-sexual, incluso en lo referente a sus propias experiencias, y algunas organizaciones que apoyaban al alumnado LGBT en colegios y universidades decidieron interrumpir su actividad ${ }^{37}$. Gracias

32 Ley Orgánica 2/2006, de 3 de mayo, de Educación, Boletín Oficial del Estado, n. ${ }^{\circ}$ Io6, de 4 de mayo de 2006.

33 Ley Orgánica 8/2013, de 9 de diciembre, para la mejora de la calidad educativa, Boletín Oficial del Estado, n. ${ }^{\circ} 295$, io de diciembre de 2013.

34 Colás Bravo, Pilar: «La construcción de la identidad de género: Enfoques teóricos para fundamentar la investigación e intervención educativa», Revista de Investigación Educativa, $25, \mathrm{n} .{ }^{\circ}$ I (2007), p. I52.

35 https://www.glsen.org/.

${ }_{36}$ www.stonewall.org.uk/.

37 Gillan, Audrey: «Section 28 gone... but not forgotten», The Guardian, 17 de noviembre de 2003, 28, http://www.theguardian.com/politics/2003/nov/17/uk.gayrights. 
en gran medida a la presión de grupos como Stonewall, el artículo fue eliminado de la legislación escocesa en 2000 y en el resto del Reino Unido en 2003. Esta organización, autodefinida como «lobby» LGBT, lleva a cabo campañas políticas y públicas dentro y fuera del ámbito escolar, siendo el cambio legislativo y la transformación de las instituciones educativas sus prioridades. Actualmente, esta organización está promoviendo una campaña contra el acoso escolar llamada «\#NOBYSTANDERS», dirigida al alumnado testigo de incidencias de acoso homofóbico, animándolo a participar de forma activa en la defensa de las víctimas.

Stonewall ha sido también uno de los colectivos participantes en el proyecto $N o$ Outsiders, una iniciativa pensada para abordar la igualdad LGBT como parte de un programa de igualdad más amplio, que tuvo lugar en Inglaterra entre 2006 y 2008 y fue financiado por el Economic and Social Research Council (ESRC). Bajo el asesoramiento de colectivos como Stonewall o Schools Out (una asociación de profesorado LGBT), profesorado de primaria e investigadores universitarios colaboraron para estudiar las formas en que la homofobia y la transfobia se sustentaban a través de acciones conscientes e inconscientes, premisas inexploradas, silencios y exclusiones tácitas. Después, el equipo investigador desarrolló, aplicó y evaluó propuestas de enseñanza que exploraban temas como la diversidad familiar o la disconformidad de género en formas que eran pertinentes y accesibles para niños y niñas ${ }^{38}$.

Para algunos profesores participantes en el proyecto, y para los colectivos LGBT que apoyaron su trabajo, el proyecto ha sido activista en su manera de visibilizar un colectivo marginalizado, sobre todo en el ámbito escolar. En las palabras de Andy, un profesor que se identificó como homosexual, «las personas homosexuales, en realidad, están en todas partes... excepto en el Currículo Nacional o, de forma visible, en nuestras escuelas» ${ }^{39}$.

En el Estado español la Federación Estatal de Lesbianas, Gais, Transexuales y Bisexuales (FELGTB) es la organización que ha tenido la mayor incidencia en el ámbito educativo. A diferencia de Stonewall en Inglaterra y GLSEN en los Estados Unidos, se define como una federación más que como un colectivo centralizado desde su formación en $1992^{40}$. Sus aproximadamente 50 asociaciones comparten y coordinan tres grandes líneas de acción:

- la reivindicación de la igualdad legal y el respeto social hacia la diversidad afectivo-sexual,

- la sensibilización y la denuncia, en las que se engloba la celebración anual Orgullo Estatal LGTB y

- la formación de las y los activistas de sus asociaciones miembro ${ }^{41}$.

${ }_{38}$ DePalma, Renée y Atrinson, Elizabeth: Undoing Homophobia in Primary Schools, Stokeon-Trent, Trentham Books, 20IO; DePALma, Renée y ATKInson, Elizabeth: Interrogating heteronormativity in primary schools: The work of the No Outsiders project, Stoke-on-Trent, Trentham Books, 2009.

39 DePalma y Atkinson: Undoing Homophobia in Primary Schools, p. XI.

$4^{\circ}$ Fue constituido como Federación Estatal de Gays y Lesbianas, con unos cambios en su denominación en los años siguientes.

${ }_{41} \quad$ Véase su página web, http://www.felgtb.org/. 
Cuenta con un área de educación dedicada a «combatir la LGTBfobia en las aulas» que se sostiene sobre dos pilares: por un lado, el apoyo y defensa del alumnado en riesgo de ser víctima de acoso escolar y, por otro, la educación en diversidad afectivo-sexual en las aulas con un enfoque proactivo, es decir, entendiendo esta como una intervención preventiva. Entre sus múltiples acciones en este campo, podemos destacar la campaña «Vuelta al cole». Como parte de esta iniciativa, se ha creado una página dirigida a alumnos, familias o profesionales que proporciona una gran gama de recursos, desde contactos y consejos para alumnado que ha sufrido acoso LGTBfóbico hasta recursos educativos que incluyen libros infantiles. De manera similar a la campaña antes comentada de la organización Stonewall, \#NOBYSTANDERs, en esta también se contempla a los testigos como actores clave en incidencias de acoso, reservando un apartado dirigido a ellos y animándolos a implicarse: «Actúa, el silencio te convierte en cómplice». Quizás lo más destacable es el apartado dirigido a las propias personas acosadoras, bajo el lema «Hago Bullying?... Deja de hacerlo inmediatamente siguiendo estos pasos». La información contemplada para este colectivo enfatiza el sufrimiento que el acoso produce a quienes lo padecen y las repercusiones legales que podría tener para quienes lo practican.

En resumen, una gran parte del trabajo educativo de la FELGTB parece girar en torno a la investigación sobre ámbitos escolares, especialmente sobre el acoso, y la publicación de guías de buenas prácticas, que tratan de concienciar al profesorado y recomendar recursos, como cuentos o libros, y algunas propuestas didácticas ${ }^{42}$. Su intervención directa en aulas o en la formación del profesorado parece más limitada, aunque en el Informe Anual de COGAM, una de las entidades fundadores de la FELGTB, figuran 484 charlas en centros educativos sobre diversidad afectivo-sexual ${ }^{43}$. También es importante destacar las organizaciones asociadas con la FELGTB que representan colectivos particulares, como la Fundación Daniela, que reconoce «una importante carencia de formación específica por parte de estos profesionales ya sea en el ámbito de la intervención médica, psicológica, social, legal o educativa» ${ }^{44}$.

Hay voces, sin embargo, que se escuchan con menos fuerza en estos movimientos LGBT, tanto dentro como fuera de las escuelas. Por ejemplo, Trujillo habla de la invisibilidad de las lesbianas en colectivos supuestamente mixtos ${ }^{45}$. También podemos señalar la escasa presencia del colectivo bisexual. La invisibilización es una de las principales vías para la subordinación, situación fácilmente atribuible a las personas que no declaran una orientación sexual definida y exclusiva:

42 Pichardo Galán et al.: op. cit.; Sánchez Saínz, Mercedes: Cómo educar en la diversidad afectiva sexual y personal en educación infantil, Madrid, La Catarata, 20ıо; Platero Méndez, Lucas: Herramientas para combatir el bullying homofóbico, Madrid, Talasa, 2008 (2. ${ }^{a}$ ed.).

43 En la página sobre «Guías de implementación de los talleres impartidos por EducaciónCOGAM en centros educativos» los detalles no parecen estar disponibles.

${ }_{44}$ Véase http://www.fundaciondaniela.org/.

45 Trujillo Barbadillo, Gracia: «La protesta dentro de la protesta. Activismos queer/cuir y feministas en el I5M», Encrucijadas. Revista Crítica de Ciencias Sociales, I2 (2016), pp. I-I8. 
La bisexualidad se convierte en una sexualidad aún más subalterna que la propia homosexualidad, despreciada por los heterosexuales que les considerarán unos depravados o depravadas; y los homosexuales les verán como unos gais o lesbianas que aún no han asumido o que no se atreven a asumir su verdadera identidad ${ }^{46}$.

De hecho, en los ámbitos escolares -sobre todo en las etapas iniciales- resulta particularmente difícil abordar las experiencias de diversidad afectivo-sexual que no se ajustan a una representación fija y estable ${ }^{47}$. La tendencia de tratar la orientación sexual en términos de familias homoparentales, por ejemplo, borra experiencias como la bisexualidad, que se escapan de las representaciones relacionales, $y$, por supuesto, excluyen a las personas que optan por no tener hijos o incluso por no formar parejas. En el siguiente apartado, examinamos con más detalle algunos otros casos que desafían los esfuerzos escolares de inclusión basados en reclamaciones identitarias.

\section{Activismo intersex y movimientos transfeministas}

Tal y como comentamos en la introducción, hemos reservado un apartado específico para analizar el activismo intersex y transfeminista, porque estos movimientos han servido de punto de inspiración para lo que se denomina una pedagogía queer o trans*gresora. Las personas con cuerpos que exceden el binomio sexual han estado sujetas a la opresión a lo largo de los tiempos, empezando con la práctica romana de arrojar al río a recién nacidos malformados (anaperon) o monstruosos (teras), prácticas encaminadas a eliminar la amenaza de contagio al resto de la población ${ }^{48}$. Las prácticas de purga no desaparecerán en los siglos posteriores, pero pasarán a las manos de la medicina, que se dedica a recortar el cuerpo del bebé con ambigüedad genital hasta que se conforme al «dimorfismo sexual, esencialismo biológico, dualidad de géneros y prácticas sexuales reproductivas» del régimen biopolítico social ${ }^{49}$.

La activista intersex Cheryl Chase compara las múltiples intervenciones «reparativas» a que las personas intersex están sometidas con la práctica cultural de la clitorectomia (circuncisión femenina), fuertemente criticada por nuestras sociedades occidentales ${ }^{50}$. Además, estas cirugías que se aplican a niñas con lo que la medicina califica como «hipertrofia del clítoris» tienen efectos secundarios

${ }^{46}$ Moreno Sánchez, Ángel y Pichardo Galán, José Ignacio: «Homonormatividad y existencia sexual. Amistades peligrosas entre género y sexualidad», AIBR. Revista de Antropología Iberoamericana, I, n. ${ }^{\circ}$ I (2006), p. I49.

47 EpsteIn, B. J.: Are the Kids All Right?Representations of LGBTQ Characters in Children's and Young Adult Literature, Bristol, Hammeron Press, 2013.

${ }_{48}$ García López, Daniel: «Politización de la vida y medicalización de la política: la producción del cuerpo intersexual», EIKASIA Revista de Filosofía, 75 (2017), pp. I4I-I57.

49 Ibidem, p. I47.

so Chase, Cheryl: «Hermaphrodites with Attitude: Mapping the Emergence of Intersex Political Activism», GLQ: A Journal of Lesbian and Gay Studies, 4, n. ${ }^{\circ}$ (1998), pp. I89-2II. 
parecidos a los producidos por ciertas formas de ablación del clítoris -como fuertes dolores o la disminución de la capacidad de experimentar placer sexual-, todo ello sin consentimiento por parte de un sujeto todavía en su primera infancia, y motivado (aunque sea con intenciones nobles) por la presión social y no por criterios de salud:

Tales prácticas médicas no hacen más que avalar y reproducir las normas sociales sobre el sexo y el género, pues se orientan fundamentalmente a «forzar» los cuerpos «ambiguos» a las dos casillas excluyentes legitimadas por la sociedad occidental (hombre/mujer) $)^{\text {s. }}$.

El concepto de biología que se maneja en estos casos, que el activismo intersex resiste y combate, asume el sexo biológico como una condición establecida en el momento de nacimiento, un binomio (hombre-mujer) claro e inmutable, que nunca falla en su asignación inicial y que debe ser suficiente para expresar la identidad de género (por supuesto, coherente y debidamente limitada) de cualquier individuo.

De modo parecido, este concepto empobrecido del sexo biológico se queda corto a la hora de explicar las experiencias de las personas que se identifican como trans. Según la definición del activista y abogado Stephen Whittle ${ }^{52}$, trans «engloba [desde] la incomodidad con las expectativas de rol, identidades queer, travestismo ocasional o más frecuente, travestismo permanente y vivido... hasta la utilización de intervenciones médicas como, por ejemplo, la terapia hormonal o quirúrgica de reasignación de género». En el Estado español, hay una tendencia a utilizar el término transexual y asociarlo estrechamente con intervenciones quirúrgicas, incluso en plataformas que reclaman derechos y reconocimiento para el colectivo. El uso del término trans* (con un asterisco) implica una visión más amplia y a la vez más crítica con respecto a los conceptos simplistas y jerárquicos del binomio. Trans* es

un concepto "paraguas» que puede incluir diferentes expresiones e identidades de género... Lo que el asterisco añade es señalar la heterogeneidad a la hora de concebir el cuerpo, la identidad y las vivencias que van más allá de las normas sociales binarias impuestas ${ }^{53}$.

Dentro de la comunidad LGBT, las personas trans* son las que sufren más discriminación, acoso, o violencia; una de cada dos personas trans* encuestadas por la FRA en 2012 fueron víctimas de violencia física, amenaza, o insulto ${ }^{54}$. Las

Si Lameiras Fernández, María; Carrera Fernández, María Victoria y Rodríguez Castro, Yolanda: El clitoris y sus secretos, Vigo, Unidade de Igualdade, Universidade de Vigo, 2013, p. 19.

52 Whittle, Stephen: «Foreword», en Stryker, Susan y Whittle, Stephen (eds.): The transgender studies reader, London, Routledge, 2006, p. xi.

53 Platero Méndez, Lucas: Trans*sexualidades: Acompañamiento, factores de salud y recursos educativos, Barcelona, Bellaterra, 20I4, p. I6.

${ }_{44}$ Véase la página trans de ILGA Europe: https://www.ilga-europe.org/what-we-do/our-advocacy-work/trans-and-intersex/trans. 
personas trans* incitan un tipo de rabia especial que busca reforzar un patriarcado que se ve amenazado. Para las mujeres trans*, el acto de rechazar el privilegio masculino que se les otorga en el momento de nacimiento se construye como un reto a la supremacía masculina, y las deja expuestas a la burla, primero por ser hombres endebles e inadecuados, y luego por no cumplir con las expectativas absurdas de feminidad a que todas las mujeres están sujetas 55 . En referencia al asesinato en 2007 del joven trans* Roberto González Onrubia, Platero ve detrás de estos crímenes violentos y sangrientos la deshumanización que conlleva su falta de inteligibilidad social: «Si no podemos distinguir el sexo de una persona, tenemos grandes dificultades para considerar que una persona sea humana; por tanto, el "cambio de género" puede suscitar un temor primordial a lo monstruoso, de pérdida de la humanidad ${ }^{56}$.

En nuestro análisis, el activismo trans* está incluido dentro del apartado sobre activismo LGBT, y también en este, porque pensamos que se pueden identificar dos líneas de acción por parte de sus activistas, asociaciones o grupos de presión: una que se dedica a asegurar el reconocimiento de los derechos básicos de los colectivos históricamente excluidos de la sociedad y la otra que pretende retar, desmentir o ampliar los conceptos básicos que tenemos sobre sexo y género ${ }^{57}$. En España, por ejemplo, la Asociación Española de Transexuales describe así sus objetivos en la página web de la organización:

Apostamos por la normalización social de la transexualidad, la lucha contra toda discriminación por motivos de orientación sexual e identidad de género. Contamos con programas de inserción sociolaboral, de prevención de salud e igualdad, asesorando a las administraciones públicas en la elaboración de leyes y políticas inclusivas; participamos junto a las universidades en estudios científicos ${ }^{58}$.

Mientras tanto, otros movimientos más radicales adoptan la filosofía de la disidencia y resistencia; como la establecida por el Manifiesto Para La Insurrección Transfeminista, que se define como protesta política y también conceptual, transformando una declaración de Simone de Beauvoir en un foco de acción:

Dinamitemos el binomio género y sexo como práctica política. Sigamos el camino que empezamos, «no se nace mujer, se llega a serlo», continuemos desenmascarando las estructuras de poder, la división y jerarquización. Si no aprendemos que la diferencia hombre mujer, es una producción cultural, al igual que lo es la

"SERANO, Julia: Whipping girl: a transsexual woman on sexism and the scapegoating of femininity, Emeryville, Seal Press, 2007.

56 Platero Méndez, R. Lucas: «¿Dónde está la ira trans*? El asesinato de Roberto González Onrubia», en Mérida Giménez, Rafael M. (ed.): Masculinidades disidentes, Barcelona, Icaria, 20ı6, p. 238.

57 Carrera Fernández, María Victoria; DePalma, Renée y Lameiras Fernández, María: «Sex/Gender Identity: Moving beyond Fixed and "Natural" Categories», Sexualities, I5, n. 8 (2012), pp. 995-10I6.

${ }_{58}$ http://transexualia.org/. 
estructura jerárquica que nos oprime, reforzaremos la estructura que nos tiraniza: las fronteras hombre/mujers9.

Estas dos líneas de trabajo no deberían ser, para nada, contrarias, aunque algunos conceptos fundamentales parecen incompatibles. Mientras Transexualia aboga por la normalización, el discurso del Manifiesto Para La Insurrección Transfeminista resiste la propia noción de normal, ampliando las críticas feministas del patriarcado al incluir la propia construcción social del binomio sexual. Se entiende que este concepto supone una barrera para la desarticulación de los discursos que imponen la desigualdad de género, ya que en cierto modo legitima esta discriminación sobre la base de diferencias dadas por naturaleza -presumiblemente indiscutibles e insalvables-. Así pues, esta concepción del sexo opera, en última instancia, a favor del mantenimiento del sistema patriarcal. Persiste también, de manera similar, un problema identificado como "homonormatividad», que surge del seno de los movimientos, y se define como «el constructo cultural que convierte a la homosexualidad en un espacio normativizado de disidencia sexual, que asume el género como elemento generador de relaciones, prácticas, e identidades sexuales y complementa la heteronormatividad a pesar de ponerla en cuestión ${ }^{60}$.

Espineira y Bourcier identifican los orígenes del movimiento transfeminista en Francia con la formación del colectivo Outrans en los años I990, y con su declaración formal Transfeminisms en 2009; en España, se originó con la incorporación del movimiento trans* a un congreso estatal feminista en el año $2000^{61}$. Según Platero y Ortega Arjonilla, los movimientos feministas y transfeministas en España gozan de una larga trayectoria de colaboración ${ }^{62}$, frente a la tendencia de confrontación característica a los mismos en el ámbito anglosajón ${ }^{63}$; esto es así gracias a la participación de mujeres trans* en conferencias feministas desde I993, la inclusión temprana de sus necesidades en la agenda compartida y en el debate abierto sobre ellas. Además, su incorporación plena al incipiente movimiento de liberación homosexual durante la década de 1980 se demoró -dada la habitual asociación en el imaginario colectivo detrans* y prostitución-debido a la preocupación de estos primeros activistas con la normalización de la homosexualidad.

De todos modos, dentro y fuera del Estado español, el movimiento transfeminista no deja de ser una extensión de los desafíos feministas hacia un mundo fuertemente patriarcal. Si bien Simone de Beauvoir propone la desnaturalización

59 Se encuentra el manifiesto, firmado por Red PutaBolloNegraTransFeminista, en diversas páginas web, como es el caso de https://ladyfestmadrid.wordpress.com/2010/or/.

60 Moreno Sánchez, Ángel y Pichardo Galán, José Ignacio: op. cit., p. isi.

${ }_{61}$ Espineira, Karine y Bourcier, Marie-Hélène/Sam: «Transfeminism Something Else, Somewhere Else», TSQ: Transgender Studies Quarterly, 3, n. ${ }^{\circ}$ I-2 (20I6), pp. 84-94.

${ }_{62}$ Platero Méndez, R. Lucas y Ortega Arjonilla, Esther: «Building coalitions: The interconnections between feminism and trans* activism in Spain», Journal of Lesbian Studies, 20, n. ${ }^{\circ}$ I (20I6), pp. 46-64.

63 STRYKer, Susan y BeTtCher, Talia M.: «Introduction Trans/Feminisms», TSQ: Transgender Studies Quarterly, 3, n. ${ }^{\circ}$ I-2 (2016), pp. 5-I4. 
del género con la publicación de Le deuxième sexe $e^{64}$, Judith Butler ${ }^{65}$ pone en duda la naturalidad del propio sexo, argumentando que la misma categoría de «natural» es un constructo ideológico, y que no es posible entender el sexo como preexistente o desligado de los discursos de género. Presenta la construcción de la identidad de género como un proceso de «asunción de un sexo», es decir, como la identificación -en alto grado forzada- con determinadas normas reguladoras que delimitan las categorías hombre y mujer y su repetición. Se entiende así la construcción de la identidad de género como un acto performativo, una representación en la que el poder constitutivo del discurso reside en su reiteración. Esta construcción es también un proceso temporal, a través del curso de la reiteración de normas el sexo se produce pero también se desestabiliza ${ }^{66}$.

La asunción (o no) de estas normas reguladoras determina la inclusión o exclusión en tales categorías. Estas categorías responden a una comprensión de la realidad articulada a través de conceptos binarios: natural/construido, sexo/ género, hombre/mujer, heterosexual/homosexual, cisgénero/transexual, nor$\mathrm{mal} /$ anormal, etc. Además, en este sistema binario las categorías aparecen como jerarquizadas, privilegiando a las personas que pertenecen a determinadas categorías -hombre, heterosexual, cisgénero-y relegando a los excluidos -mujer, homosexual, transexual- a una posición subalterna y marcada por el estigma social.

Butler expone a lo largo de su obra la idea de la heterosexualidad obligatoria, que configuraría mediante estas exclusiones un sistema social heteropatriarcal, donde los mayores privilegios están reservados a los cuerpos expresados y leídos como masculinos y con deseos heterosexuales ${ }^{67}$. Sin embargo, el concepto de performatividad del género y el hecho mismo de que la vida en los márgenes -precarizada y marcada por el estigma, pero vida al fin y al cabo- sea posible, perfila una ventana de oportunidad. Existe así todo un abanico de sujetos trans*gresores -tomando el concepto paraguas de Platero- ${ }^{68}$ que de diversas formas transgreden $y$, de algún modo, fallan en reproducir y conformar las normas reguladoras del sistema binario sexo/género: lesbianas, gais y bisexuales, personas transgénero, hombres y mujeres que no se ajustan al ideal corporal que la matriz heterosexual impone, etc. ${ }^{69}$. Estos fallos abren profundas grietas en la aparentemente sólida fachada del sistema binario hegemónico, poniendo de manifiesto el carácter artificial de dichas normas y generando espacios de rearticulación de las mismas.

${ }^{64}$ De Beauvorr, Simone: Le deuxième sexe, Paris, Gallimard, 1949.

65 Butler, Judith: Cuerpos que importan: sobre los limites materiales y discursivos del "sexo", Barcelona, Paidós, 2002.

${ }_{66}$ Ibidem, p. 29.

${ }_{7}$ Ibidem.

68 Trans"sexualidades: Acompañamiento, factores de salud y recursos educativos.

69 DePalma, Renée: «O que se pode aprender dos corpos transgresores», en González Penín, Anabel; López Díaz, Ana Jesús y Aguayo Lorenzo, Eva: Trazos de xénero no século XXI: Actas, III Xornada Universitaria Galega en Xénero, Vigo, Universidade de Vigo, 20I5, pp. I49-I64; Berná, David; Cascone, Michele y Platero Méndez, Raquel (Lucas): «¿Qué puede aportar una mirada queer a la educación? Un estado de la cuestión sobre los estudios sobre la LGTBfobia y educación en el Estado español», The Scientific journal of Humanistic Studies, 6, n. ${ }^{\circ}$ (2012), p. 22. 


\section{La pedagogía queer/cuir o trans*gresora}

En el capítulo «El género en llamas: cuestiones de apropiación y subversión», Butler lanza una pregunta provocadora que invita a una respuesta tanto desde el activismo como por parte de la educación: «¿Hay otras maneras de ser interpelado y constituido por la ley? ¿Hay otras maneras de ocupar la ley y ser ocupado por

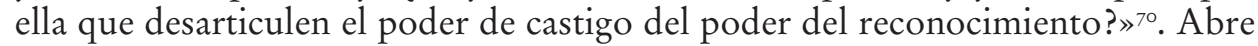
con este interrogante una «gama de desobediencias» que van desde el rechazo a la rearticulación y la reapropiación.

El propio término queer es un ejemplo de esa rearticulación. Queer es un término inglés que significa literalmente «raro», «extraño», «anómalo», y era empleado como insulto homofóbico contra aquellos individuos cuyo deseo sexual no conformaba la norma heterosexual, poniendo de manifiesto el carácter desviado de su sexualidad. Sin embargo, el término vive una reapropiación por parte de colectivos politizados, resignificándose y transformándose en un estandarte de irreverencia y alteridad orgullosa. Al mismo tiempo, activistas como Gracia Trujillo consideran que sus propias raíces en el contexto anglosajón conllevan una perspectiva geopolítica y, por lo tanto, prefieren utilizar el término latinizado cuir o el compuesto queer/cuir: «Para muchxs activistas (entre las cuales me incluyo) seguir utilizándolo es también una forma de reconocer la trayectoria política de la que venimos, sin dejar de considerar las críticas al término, que además comparto: queer en algunos contextos (como el de América Latina, pero no sólo) resuena como algo anglosajón y de élite académica, de ahí la necesidad de descolonizar el término» ${ }^{71}$.

Habitualmente, en España se identifica como el primer texto en el que se reflexiona sobre una posible relación entre teoría queer/cuir y educación el artículo de Deborah P. Britzman «La pedagogía transgresora y sus extrañas técnicas» ${ }^{72}$. En él critica el modelo educativo tradicional que se viene desarrollando desde la Modernidad, «sin una teoría del conflicto y de la otredad», y se interroga acerca de las posibilidades de una pedagogía preocupada por su intervención en la producción social de los individuos, que sea crítica con el concepto de normalidad y capaz de «transgredir las identidades estables» o cerradas ${ }^{73}$.

¿Puede la escuela contribuir a esa desestabilización de las identidades? ¿Qué prácticas pueden facilitar esas «desidentificaciones colectivas» con las normas reguladoras del heteropatriarcado que propone Butler? ¿Como educadores, cómo promovemos modos de subjetivarnos que derriben las barreras que separan el yo de esos otros «extraños»? ¿Cómo alentamos formas de ser que no precisen rechazar la diferencia para reafirmar la propia identidad?

70 Butler, Judith: op. cit., p. I8o.

${ }_{71}$ Trujlllo Barbadillo, Gracia: op. cit., p. 5.

72 Britzman, Deborah P.: «La pedagogía transgresora y sus extrañas técnicas», en MÉRIDA Jiménez, Rafael M. (ed.): Sexualidades transgresoras. Una antología de estudios queer, Barcelona, Icaria, 2002, pp. 197-228. En su versión original, su artículo «¿Hay una pedagogía queer? O, no leas tan recto», se publicó tres años antes, pero no fue traducido al español hasta 2016.

73 Ibidem, p. 198. 
No existen manuales de instrucciones o recetas mágicas para una praxis educativa trans"gresora; con todo, consideramos que del activismo transfeminista se pueden extraer varias claves para planificar una intervención educativa.

I. Perspectiva interseccional: se trata de un concepto fundamental para una pedagogía queer/cuir o trans*gresora. La teoría postfeminista -en la que se encuadra la Teoría Queer- pretende superar una de las críticas más constantes en la Historia de la teoría feminista: la falta de atención a otras categorías a través de las que operan otras formas de opresión como son la clase, la raza o la diversidad funcional ${ }^{74}$.

2. Entender la alteridad: implica realizar una crítica activa a la escuela tradicional como uno de los primeros espacios de ensayo de las prácticas sociales de control, vigilancia y penalización de las identidades no hegemónicas. Desde esta perspectiva, se entiende la identidad como el reconocimiento de «mismidades y otredades» ${ }^{75}$, que nos sitúan y sitúan a los demás como integrantes o excluidos de determinadas categorías, grupos sociales o colectivos; y exige tener siempre presentes las implicaciones de esas inclusiones y exclusiones en términos de derechos y privilegios ${ }^{76}$.

3. La ruptura con el sistema binario: la pedagogía trans*gresora tiene como objetivo desnaturalizar y desestabilizar los binomios sexo/género y normal/anormal, que constituyen dos importantes pilares de la heteronormatividad, por lo que no se limitará a incorporar discursos y modelos sensibles hacia la diversidad sexual y de género, sino que procurará la reflexión activa del alumnado acerca de estas categorías, de los contextos en los que operan y de las exclusiones que generan. Perseguirá también otros binarismos -que funcionan como categorías cerradas, opuestas y jerarquizadas- profundamente enraizados en el saber occidental, como verdadero/falso, colonizado/incivilizado, cuerpo/ mente, etc. ${ }^{77}$.

4. La atención al cuerpo simbólico: siguiendo de nuevo a Butler, si el cuerpo sexuado no es pura materia, sino que se materializa atravesado y limitado por los discursos de género, cabe entonces incidir en este proceso de materialización, educar el cuerpo ${ }^{78}$. Entrevemos así una dimensión del cuerpo que no es puramente material, en la que podemos «leer» cuestiones que trascienden lo biológico, y que puede hablarnos de género, raza, clase, etc. ${ }^{79}$. Según Galarte, el cuerpo trans sirve de vehículo para desvelar y entender la producción social de género: «Situar el cuerpo trans en el centro de la producción de conocimiento es

74 Alegre Benítez, Carolina: «La perspectiva postfeminista en educación. Resistir en la escuela», Revista Internacional de Investigación en Ciencias Sociales, 9, n. I (2013), p. I49; Berná, Cascone y Platero Méndez: op. cit., p. iz.

75 Berná, Cascone y Platero Méndez: op. cit., p. I2.

76 Britzman, Deborah P.: op. cit.

77 Alegre Benítez, Carolina: op. cit., p. I49; Britzman, Deborah P.: op. cit., pp. 198-200.

78 ButLer, Judith: op. cit.

79 Planella Ribera, Jordi y Pie Balaguer, Asun: «Pedagoqueer: Resistencias y Subversiones Educativas», Educación XXI, I5, n. ${ }^{\circ}$ I (20I2), pp. 265-283. 
una transpedagogia imprescindible [...]; este tipo de transpedagogia es radical en la medida en que critica, y que tiene la capacidad de transformar, como la autoridad y el poder construyen y organizan el conocimiento» ${ }^{80}$.

5. Superar las posturas esencialistas: la pedagogía trans*gresora busca ir más allá de los discursos de la tolerancia o de la inclusión. Pretende superar la mera visibilización o las posiciones esencialistas que se limitan a informar sobre la existencia de identidades disidentes; procura un discurso donde las personas vayan por delante de las etiquetas, que ponga en valor la diversidad, que legitime las formas de vida disidentes y en el que las protagonistas trans*gresoras no aparezcan victimizadas, sino como modelos empoderadores y agentes de cambio $^{8 \mathrm{r}}$.

6. De las calles a las aulas: la pedagogía trans*gresora se caracteriza por el empleo de estrategias propias de la política y el activismo queer. Poner de manifiesto el carácter performativo del género -de la propia identidad, en cierto modo-a través de la teatralidad, de la hipérbole paródica ${ }^{82}$; llevar a extremos ridículos mandatos sociales, prácticas o leyes encorsetadas y excluyentes; aplicar en las aulas lo que siguiendo a Mikhail Bakhtin ${ }^{83}$ podríamos llamar una política de lo carnavalesco, de la resignificación y la transformación, del orgullo de la diferencia, del constante cuestionamiento de lo establecido que posibilita la vida en los márgenes del orden social ${ }^{84}$.

En lo relativo a las contribuciones de los movimientos activistas a la práctica escolar, las colaboraciones que dan lugar a una transpedagogía son más escasas, incluso en el plano internacional. Podemos ver una excepción volviendo al ejemplo de proyecto No Outsiders en Inglaterra. Uno de los colectivos que participaban en la iniciativa fue la organización Gendered Intelligence ${ }^{85}$, que se compromete con la idea de que todo el mundo puede ser inteligente con respecto al género. Su papel, en principio, se iba a limitar a un taller sobre realidades trans* dirigido al profesorado participante, para ayudarles a encontrar formas de incluir estas realidades en la práctica docente. Como resultado, algunos de estos profesores y profesoras decidieron después invitar a su codirector, Jay Stewart, en tanto que hombre trans, activista y educador social, a trabajar directamente con el alumnado en las aulas. I47.

so Galarte, Francisco J.: «Pedagogy», TSQ: Transgender Studies Quarterly, I, n. ${ }^{\circ}$ I-2 (20I4), p.

${ }_{8}$ Berná, Cascone y Platero Méndez: op. cit.; DePalma, Renée y Atkinson, Elizabeth: «Beyond tolerance: Challenging heteronormativity in primary schools through reflective action research», British Educational Research Journal, 35, n. ${ }^{\circ} 6$ (2009), pp 837-855.

${ }_{82}$ Un ejemplo de estos cruces entre humor y activismo popular es un segmento del programa LastWeekTonight with John Oliver, dedicado a derechos de personas trans; véase https://www. youtube.com/watch?v=hmoAX 96 MOc. Otro es el monólogo cómico del activista estadounidense Rikki Wilchins, Themangina Monologues: A OneTrans Show; véase https://www.youtube.com/ watch?v=82 UU 5 JEI2ZM.

$8_{3}$ Bakhtin, Mikhail: Rabelais and his world, Bloomington, Indiana University Press, 1984.

84 Carrera Fernández, DePalma y Lameiras Fernández: op. cit.

85 Véase http://genderedintelligence.co.uk/. 
Stewart empezó su trabajo con una actividad diseñada para desvelar nuestra propia implicación en la atribución de género. Mostrando una serie de fotos de personas, preguntó si eran hombres o mujeres, niñas o niños, y animó a los alumnos a explicar y defender sus conclusiones. En las entrevistas que los investigadores de No Outsiders llevamos a cabo con él como parte del proyecto, reflexionó sobre los objetivos y los resultados de su intervención. Para Stewart, el hecho de que los alumnos estuvieran seguros de sus interpretaciones, pero al mismo tiempo tomasen conciencia de que no era tan fácil explicar sus razones, fue un paso primordial para entender el concepto complejo de la construcción social del sexo-género. Luego repitió la actividad con fotos de juegos, para abrir un debate sobre características (por ejemplo, colores) que se asocian con niñas o niños. Finalmente, cada participante elaboró un autorretrato multimedia que respondía a cuatro preguntas. Stewart acuñó los términos, en inglés, girlishgirl y boyishboy para comunicar a los niños de forma comprensible la conformidad a las expectativas de género, para luego poder discriminar sus experiencias y preferencias de las que se esperaban de ellos. Hemos traducido estas frases aquí usando «tipo niña» o «tipo niño»:

¿Cómo percibes tu propio género?

¿Eres una niña «tipo niña» o un niño «tipo niño», o eres un poco de ambas cosas? ¿Qué es lo que haces que alguna gente pueda considerar «tipo niño» o «tipo niña»? ¿Qué es lo que te gusta sobre ser niña, niño, niña «tipo niño» o niño «tipo niña»?

Cada retrato empezó con la frase Cuando nací, fui asignad@ , que el alumnado tenía que completar. En palabras de Stewart: "Quería captar ese momento: es un niño, o es una niña. ¿YY por qué es tan importante? Está directamente relacionado con la cultura porque tienes que saber qué nombre le deberías dar, y tienes que saber cómo vestir a ese bebé. ¡Si no, se puede confundir y sería un desastre! (risa)».

En la intervención con los alumnos del aula de $6 .^{\circ}$, decidió dar un paso más de lo que había previsto. Cuando todo el mundo había compartido su trabajo con los compañeros, Stewart leyó en voz alta la primera frase de su autorretrato multimedia: «Cuando nací, fui asignado niña». En ese momento, los niños se enteraron de su experiencia trans*, y de cómo las vivencias del propio Jay se comparaban con sus expectativas acerca de la experiencia de las personas trans*. Según Stewart, no había planificado al principio revelar su historia femenina, pero vio la oportunidad, de forma espontánea, de hablar sobre sus experiencias, el tipo de intervenciones quirúrgicas y hormonales disponibles y las decisiones tomadas en su caso. Comentó después: «Esto es mi trabajo. Decir que trans* es un espectro que trata de muchas cosas, no exclusivamente de la cirugía. Nuestro entendimiento sobre lo trans* está limitado por las representaciones en los medios, obsesionados con la cirugía» ${ }^{86}$.

86 Para un análisis más pormenorizado del trabajo de Stewart en las aulas en el marco del Proyecto No Outsiders, véase DePalma, Renée: «Choosing to lose our gender expertise: queering sex/ gender in school settings», Sex Education, I4 (20II), pp. I-I5. 
Otras aplicaciones de los movimientos transfeministas se enfocan en el uso de la literatura infantil y juvenil para explorar experiencias de protagonistas trans ${ }^{*}$ gresoras ${ }^{87}$. En una iniciativa estadounidense, una profesora lesbiana, miembro de un colectivo de profesores dedicado a abordar la homofobia a través de su docencia, decidió experimentar con cuentos y vídeos con protagonistas infantiles que se identifican como trans* o que simplemente transgreden expectativas de género ${ }^{88}$. Esta iniciativa nos sirve para poner en relieve dos aspectos importantes de la pedagogía trans*gresora: I) no tiene por qué estar limitada a docentes trans*, pues resulta aplicable por parte de cualquier docente concienciado y con recursos suficientes, y 2) se puede implementar desde una perspectiva interseccional, teniendo en cuenta que esta iniciativa empezó con un enfoque antirracista y se amplió sobre la marcha para incluir una mirada crítica sobre normas sociales sobre sexo y género.

En el Estado español, las perspectivas trans* están incluidas en las acciones activistas de la FELGBT y algunas de sus entidades federadas, destacando el enfoque específico de la Fundación Daniela en apoyo de los menores trans*, sobre todo en su objetivo de "formación a centros de estudios destinados tanto al equipo docente como a los alumnos». Por ejemplo, en sus materiales dirigidos a abordar y prevenir el acoso escolar, se utiliza a menudo el término inclusivo "LGTBfóbico», y las experiencias de personas trans* están recogidas en los materiales escolares recomendados por la federación. Este es el caso de la Guía didáctica sobre la transexualidad para jóvenes y adolescentes desarrollado por el COGAM en $20 \mathrm{II}^{89}$. El documento, en formato de viñetas sencillas y atractivas en las que figuran personajes adolescentes, empieza con la siguiente introducción:

Se suele decir que las personas transexuales son mujeres «presas» en cuerpo de hombres y hombres «presos» en cuerpo de mujer. La verdad es que esta definición es bastante acertada ya que podemos decir que se trata de chicas que se sienten como tales, aunque su apariencia física no sea de mujer y chicos que se reconocen como chicos, aunque tengan cuerpo de mujer (p. 4).

A pesar de su claridad y apariencia lúdica, este recurso introduce conceptos complejos como el sexo psicológico, los procesos de autosexuación y los tratamientos hormonales. Aunque la idea de que las personas trans* están atrapadas en cuerpos equivocados ha provocado resistencias y críticas por parte de colectivos trans*, y la guía presenta una visión binomial de las experiencias trans*, se pueden analizar aquí elementos fundamentales de una pedagogía trans*gresora, sobre todo en su mirada social sobre la asignación de sexo:

87 Bartholomaeus, Clare y Riggs, Damien W.: Transgender People and Education, New York, Palgrave MacMillan, 2017; Miller, sj «Trans*+ing Classrooms: The Pedagogy of Refusal as Mediator for Learning», Social Sciences, 5, n. ${ }^{\circ} 3$ (2016), p. 34.

88 Ryan, Caitlin L.; Patraw, Jasmine M. y Bednar, Maree: «Discussing Princess Boys and Pregnant Men: Teaching About Gender Diversity and Transgender Experiences Within an Elementary School Curriculum», Journal of LGBT Youth, IO, n. ${ }^{\circ}$ I-2 (2013), pp. 83-IO5.

89 Disponible en la página de la FELGTB, http://www.felgtb.org/temas/educacion/documentacion/secundaria/i/49i/448/guia-didactica-sobre-transexualidad-para-jovenes-y-adolescentes. 
Inmediatamente y de forma instintiva, sin ser conscientes de ello, lo primero que determinamos es el sexo al que pertenece [cada persona], entre otras cosas para dirigirnos a él/ella o hablar de ella en femenino o masculino... y ¿cómo lo hacemos? Pues mirando si su apariencia coincide con lo que nosotros tenemos entendido que es ser hombre o mujer (p. Io).

Además, desde fuera del ámbito de la FELGTB, están apareciendo materiales publicados para facilitar iniciativas trans* en el aula. El libro Trans*exualidades. Acompañamiento, factores de salud y recursos educativos ${ }^{9 \circ}$ ofrece herramientas prácticas en forma de actividades, materiales y recursos. La primera parte del libro está dedicada a desmentir el concepto esencialista del «sexo biológico», que excluye la construcción sociocultural de género:

Cuando se usa el concepto sexo, a menudo, se [ha] hecho alusión a aquella herencia genética que conforma a las personas con ciertas anatomías, habilidades y aspectos... En la actualidad, esta perspectiva sobre el sexo está siendo profundamente rebatida, ya que no es posible afirmar que el sexo exista previamente o separado de la cultura (p. 28).

En su segundo apartado, «Herramientas y recursos», se proporcionan ideas y materiales concretos, aprovechando una gran gama de películas, vídeos, artículos de prensa, viñetas, etc., que representan realidades trans*gresoras y su posible aplicación educativa.

Chrysalis, una asociación de familias de menores transexuales independiente, se autoidentifica en su página web como un colectivo de apoyo mutuo para familias, pero sus acciones van mucho más allá, con un claro objetivo de incidencia en los centros educativos y en la educación de la sociedad sobre el género ${ }^{91}$. Por una parte, esta línea de actuación queda patente en algunas reflexiones de su actual presidenta, Natalia Aventín Ballarín:

Sabemos que las circunstancias de especial vulnerabilidad del colectivo LGTBI+ tienen el mismo origen, la genitalidad y la ideología (de género) aplicada a ésta. Desde que al nacer, o incluso antes, se nos clasifica en base a los genitales en hombres o mujeres -la intersexualidad se medicaliza para no nombrarla- y se da por hecho que vamos a ser personas heterosexuales. Es evidente que los genitales no determinan ni condicionan la orientación sexual, como tampoco lo hacen con la identidad.

$\mathrm{Su}$ argumento principal parece centrarse en la idea de que el activismo homosexual ha conseguido, con esfuerzo y dificultad, rebatir la idea de que los genitales determinan la orientación sexual; sin embargo, esta doctrina falsa sigue determinando el imaginario colectivo sobre la identidad de género.

Por lo tanto, como Gendered Intelligence en Inglaterra, Chrysalis entiende la generalización de la educación en diversidad afectivo-sexual y de género como

9o Platero Méndez: Trans*sexualidades: Acompañamiento, factores de salud y recursos educativos.

${ }_{91}$ Véase http://chrysallis.org.es/. 
necesaria para el bienestar de las personas disidentes o de las que no conforman con esta ideología errónea. Esto no quiere decir que dejen a un lado las campañas legislativas o actos de orgullo y visibilización, pero adoptan una perspectiva claramente trans*feminista en su atención a cómo la diversa realidad corporal excede la restringida ideología social. Entre sus materiales recientes vemos el cuento Niñas y niños. Cada una, cada uno, diferente (Mayor y Monteagudo, 2016), también disponible en formato vídeo en la página ChrysalisEuskalHerria ${ }^{92}$. El libro presenta una diversidad de expresiones e identidades de género, entre las que figuran niños con vulva y niñas con pene.

\section{Conclusión}

A pesar de los datos aportados en relación al bullying homo- y transfóbico, que legitima la introducción de cuestiones relativas a la educación en la diversidad afectivo-sexual y de género en las aulas, existen numerosas trabas y resistencias sociales que dificultan enormemente esta labor. Cada contexto, dada su trayectoria sociopolítica, presenta sus particularidades. Mientras que los movimientos sociales en defensa de los derechos del colectivo LGBT se alzaron relativamente tarde en España, podemos decir que gozan de una colaboración más fuerte con las luchas feministas que en algunas otras partes del mundo. Sin embargo, pese a esta fortificante relación, la diversidad afectivo-sexual en los centros educativos españoles, de momento, carece de iniciativas específicas como las que hemos visto en otros países. Podríamos decir incluso que la política educativa estatal mantiene una posición retrógrada con respecto a la incorporación del tema en el currículo; pero, al mismo tiempo, se percibe la emergencia relativamente reciente de una gran preocupación por los ámbitos escolares - por un lado, con las iniciativas para promover el bienestar de niñas y niños transgresores, y por otro, a través de la creciente producción de recursos diseñados para ampliar y mejorar conceptos relacionados con el sexo, el género, y la sexualidad-.

En otros lugares, ya hemos visto fuertes resistencias a su inclusión en el currículo escolar (como es el caso del Christian Institute en Inglaterra). En España, aún nos queda por ver si en este escenario polarizado una colaboración incipiente entre movimientos sociales y profesorado resultará, también, en protestas organizadas por algunos sectores de la sociedad.

De momento, podemos ver los inicios de pequeños contramovimientos, altamente mediatizados ${ }^{93}$. Las críticas de los sectores más conservadores, que cristalizan en iniciativas como plataformas de rechazo a la materia de Educación para la Ciudadanía de la ya extinta Ley Orgánica de Educación (2006) -en la que se

92 Véase chrysalliseh.eus.

93 «Los puntos más polémicos de la ley de igualdad LGTB», $A B C$, I9 de septiembre de 20I7, http:// www.abc.es/sociedad/abci-puntos-mas-polemicos-ley-igualdad-lgtb-201709192225_noticia.html. 
abordaban los derechos LGBT-94, la caravana «Hazte Oír»95, etc., inducen a los sectores más progresistas a defender la relevancia de una educación que atienda el plano afectivo-sexual, aborde la desigualdad de género y cultive la sensibilidad hacia los derechos LGBT.

Así, mientras que estamos inmersos en la tarea de explicar por qué es importante hablar con los alumnos de afectividad y sexualidad, de recordar las estadísticas a quien las ignora, de justificarnos ante los ataques de los sectores más reaccionarios, «nos llevan a un pensamiento binario a favor/en contra [de la inclusión de la diversidad afectivo-sexual en el currículo] que impide tener debates productivos sobre qué educación queremos sobre la sexualidad», como señalan Berná, Cascone y Platero ${ }^{96}$. Este encasillamiento elude otras preguntas y debates importantes relativos a cómo la escuela transmite y refuerza roles, mandatos y estereotipos de género, qué orientación debemos darle a la educación afectivosexual, cómo incluimos estos aspectos en el currículo o de qué herramientas metodológicas y recursos podemos valernos para esta tarea.

Los colectivos activistas han aportado mucho al contexto educativo en términos de promoción de y participación en la elaboración de protocolos educativos, investigación sobre el acoso escolar y elaboración de materiales y guías didácticas. Se echa de menos, sobre todo, su participación más directa en actividades docentes o en la formación de profesionales. Pero este paso no depende tanto de los colectivos, que parecen estar motivados y preparados para este nivel de colaboración, como de las culturas educativas de los centros y de las facultades que forman al profesorado.

Sin embargo, pese a todas las trabas y dificultades, incluida la legislación educativa estatal, en varios Ayuntamientos y Diputaciones comienzan a desarrollarse planes de fomento de la igualdad y del respeto por la diversidad afectivo-sexual y de género que incluyen charlas o talleres en escuelas e institutos, entre otras acciones; este es el caso -por citar solo algunos- de Barcelona, Cádiz, Tenerife o el de la ciudad desde la que escribimos, A Coruña. Queda mucho camino todavía, pero podemos ser optimistas: el activismo LGBT empieza a desbordar sus propias fronteras inundando poco a poco las instituciones. Solo nos queda apostar desde aquí para que estas nuevas propuestas educativas no se dejen exclusivamente en manos de grupos empresariales que detectan aquí un nicho de mercado, y se siga contando con la infinitamente enriquecedora presencia y colaboración de los colectivos activistas.

94 «La nueva Educación para la Ciudadanía, sin "igualdad", "sexismo" o "racismo"», El Mundo.es, 4 de agosto de 2002, http://www.elmundo.es/elmundo/20I2/08/04/espana/I34407I84I.html; PAPell, Antonio: «Las seis claves de la polémica de la asignatura Educación para la Ciudadanía», Ecodiario.es, 28 de enero de 2009, http://ecodiario.eleconomista.es/espana/noticias/999466/or/o9/ Por-que-nace-la-polemica-con-Educacion-para-la-Ciudadania-Que-es-exactamente.html.

95 Galaup, Laura: «La que tiene que hacer el tránsito es la sociedad, no los niños transexuales», eldiario.es, 3 de marzo de 2017 , http://www.eldiario.es/sociedad/HazteOir-autobus_de_HazteOirtransfobia_O_618038938.html; «Un tercer autobús de Hazte Oír estaciona frente a un instituto de Alcobendas", El Mundo.es, 7 de marzo de 2017, http://www.elmundo.es/madrid/2017/03/o7/58be7 cf $7466_{3} f_{c a 308 b} 6$ bd.html.

96 Berná, Cascone y Platero Méndez: op. cit., p. 9. 